Ist ein Comet, der sich in parabolischer oder hyperbolischer Bahn dem Sonnensystem genzhert hat, durch Jupiter in eine elliptische Bahn geworfen worden, so bewirken im Allge. meinen die Jupiterstörungen selbst, dass der Comet auf die Sonne oder auf Jupiter stürt oder sich wieder in parabolischer oder hyper-

bolischer Bahn vom Sonnensystem entfernt. Tritt das ausnahmsweise nicht von selbst ein, so kann es doch zu Wege gebracht werden, indem man dem Cometen irgend wann einen beliebig kleinen Stoss ertheilt, das heisst, indem man seine osculirenden Elemente beliebig wenig ändert. «*)

München 1896 April 27.

\title{
Karl Schwarzschild.
}

*) Aehnliche Ueberlegungen, die bei späterer Gelegenheit mitg etheilt werden sollen, lassen sich auch für das allgemeine $n$-Körperproblem und auch in dem Falle, dass man es nicht mit der Bewegung eines aus sehr grosser Entfernung kommenden Körpers zu thun hat, durchfilhren.

\section{Researches on the orbit of $\eta$ Coronae Borealis $=\Sigma 1937$.}

By T.F.F. See.

This remarkable star was discovered by Sir William Herschel, Sept. 9, 1781 . It proved to be one of the first objects which gave distinct evidence of orbital motion, and the binary character of the system was fully recognized by Herschel in 1803 . Since the time of Struve the measures are both numerous and exact. The pair is always rather close, but as the components are nearly equal in magnitude it is generally easy to separate; and while the errors in angle are larger than in case of wider objects the absolute errors of the observed positions are correspondingly reduced by the shortness of the radius vector.

Numerous orbits have been published by previous computers; the following table gives a summaty of the elements which is fairly complete. It will be seen that in recent years the elements have not been changed by very large quantities, and yet they are severally by no means identical.

\begin{tabular}{|c|c|c|c|c|c|c|c|c|}
\hline$P$ & $T$ & $e$ & $a$ & $\Omega$ & $i$ & $\lambda$ & Authority & Source \\
\hline 44.242 & 1806.20 & 026034 & 0.8325 & $220: 6$ & $37^{\circ} \cdot 4$ & $358: 63$ & Herschel I 833 & Mem. R. A. S., VI.1 56 \\
\hline 43.246 & 1850.23 & 0.3376 & 1.0879 & $24 \cdot 3$ & $7 \times 13$ & $261 \cdot 35$ & Mädler 1842 & - \\
\hline $43 \cdot 310$ & 1815.20 & 0.3537 & I.1912 & 22.6 & 71.5 & 263.17 & Mädler 1842 & 一 \\
\hline 42.500 & 1807.21 & 0.289 & 0.9024 & 20.1 & 5947 & 2 I 5.2 & Mädler 1842 & - \\
\hline $42.50 \mathrm{I}$ & 1805.666 & 0.4743 & 1.0125 & 10.52 & 65.65 & 227.17 & Villarceau 1842 & - \\
\hline 66.257 & 1780.124 & 0.4695 & I. 1108 & 4.42 & 58.05 & 194.62 & Villarceau 1852 & - \\
\hline $67 \cdot 309$ & $1779.33^{8}$ & 0.4043 & 1.2015 & 9.87 & $59 \cdot 3^{2}$ & 185.0 & Villarceau 1852 & A. N. 868 \\
\hline $43.1 \times 5$ & 1850.329 & 0.2865 & 0.9567 & 22.3 & 60.67 & 215.48 & Winnecke & - \\
\hline $41 \cdot 5^{8}$ & 1850.26 & 0.2625 & 0.827 & 26.7 & $5^{8.0}$ & 211.4 & Wijkander & 一 \\
\hline $41 \cdot 576$ & 1850.26 & 0.2625 & 0.827 & 26.7 & $5^{8.0}$ & 215.6 & Dunér I 871 & A. N. 1868 \\
\hline 40. I 7 & 1849.9 & 0.287 & 0.985 & 22.2 & 60.4 & $224 \cdot x$ & Flammarion 1874 & Cat. ét. doub. 88 \\
\hline 41.562 & 1850.792 & 0.2667 & 0.892 & 25.72 & 59.68 & 218.6 & Doberck 1880 & A. N. 2338 \\
\hline 41.6 & $1892 \cdot 3$ & 0.33 & 0.86 & 26.9 & 55.0 & 220.5 & Comstock I 893 & Proc. Am. Assoc., 1894 \\
\hline
\end{tabular}

Making use of all the measures up to 1895 , we find the following elements of $\eta$ Coronae Borealis :

$$
\begin{array}{lrl}
P=41.60 \text { years } & \Omega & =27^{\circ} 10 \\
T=1892.50 & i & =58.50 \\
e=0.267 & \lambda=217.57 \\
a=0.9165 & n=+8.65
\end{array}
$$

Apparent orbit :

Length of major axis $=1.804$

Length of minor axis $=0.934$

Angle of major axis $=28: 7$

Angle of periastron $=229.0$

Distance of star from centre $=0.209$.

Comparison of computed with observed places.

\begin{tabular}{c|r|r|c|c|c|c|c|l|}
\hline$t$ & \multicolumn{1}{|c|}{$\theta_{o}$} & \multicolumn{1}{c|}{$\theta_{c}$} & \multicolumn{1}{c|}{$\rho_{o}$} & $\rho_{c}$ & $\theta_{o}-\theta_{c}$ & $\rho_{o}-\rho_{c}$ & $\mathrm{n}$ & \multicolumn{1}{|c}{ Observers } \\
\hline 1781.69 & 30.7 & $27^{\circ} .4$ & - & 1.08 & +3.3 & - & 1 & Herschel \\
1802.69 & 179.7 & 174.8 & - & 0.63 & +4.9 & - & 1 & Herschel \\
1823.27 & 25.9 & 27.3 & 1.58 & 1.08 & -1.4 & +0.50 & 2.1 & Herschel and South \\
1826.77 & 35.3 & 37.9 & 1.07 & 1.09 & -2.6 & -0.02 & 4 & Struve \\
1829.55 & 43.2 & 47.0 & 0.96 & 1.01 & -3.8 & -0.05 & 2 & Struve \\
1831.48 & 51.4 & 54.5 & 0.95 & 0.92 & -3.1 & +0.03 & 15.4 & Da. 2.0; Herschel 10.1; $\Sigma_{3}$
\end{tabular}




\begin{tabular}{|c|c|c|c|c|c|c|c|c|}
\hline$t$ & $\theta_{0}$ & $\theta_{c}$ & Qo & $\rho_{c}$ & $\theta_{0}-\theta_{c}$ & $\rho_{0}-\rho_{c}$ & $\mathbf{n}$ & Observers \\
\hline 1832.60 & $56: 9$ & $59 \div 5$ & o." 74 & 0.86 & $-2: 6$ & -0.12 & 13.5 & Herschel 9.2; Da. 1.0; $\boldsymbol{\Sigma}_{3}$ \\
\hline 1833.33 & 62.7 & 63.4 & 0.72 & 0.82 & -0.7 & -0.10 & $1 \mathrm{t} .2$ & Herschel 8.2 ; Dawes 3.0 \\
\hline 1834.84 & 69.1 & 72.5 & 0.70 & 0.73 & -3.4 & -0.03 & 1 & Struve \\
\hline $1835.4 \mathrm{I}$ & $75 \cdot 7$ & 76.6 & 0.74 & 0.70 & -0.9 & +004 & 5 & Struve \\
\hline $183^{6.52}$ & 88.8 & 85.9 & 0.56 & 0.63 & +2.9 & -0.07 & 6 & Struve \\
\hline 1839.70 & 125.9 & 122.2 & 0.63 & 0.53 & $+3 \cdot 7$ & +0.10 & 4 & Da. $2 ; O \Sigma_{2}$ \\
\hline 1840.57 & 136.0 & 133.4 & 0.51 & 0.53 & +2.6 & -0.02 & 7 & $O \Sigma_{5} ; \mathrm{Da} .2$ \\
\hline 1841.52 & 149.8 & 146.0 & $0.5^{\circ}$ & 0.54 & +3.8 & -0.04 & 16.11 & Mä. $5 ; O \Sigma_{5} ; \mathrm{Da} .6 . \mathrm{r}$ \\
\hline 1842.48 & 157.8 & $157 \cdot 5$ & 0.54 & 0.57 & +0.3 & -0.03 & 9 & Mä. $5 ;$ Da. $2 ; O \Sigma_{2}$ \\
\hline 1843.50 & 169.2 & 168.2 & $0.5^{8}$ & 0.60 & +1.0 & -0.02 & 13 & Mä. 6; Mă. 7 \\
\hline $1844 \cdot 38$ & 174.0 & 176.4 & 0.57 & 0.64 & -2.4 & -0.07 & 3 & Mädler \\
\hline $1845 \cdot 46$ & 179.3 & 184.8 & $0.5^{8}$ & 0.68 & $-5 \cdot 5$ & -0.10 & 6 & O. Struve \\
\hline 1846.61 & I $95 \cdot 7$ & 194.1 & $0.6 \mathrm{I}$ & $0.7 \mathrm{I}$ & +1.6 & -0.10 & 3 & O. Struve \\
\hline 1847.42 & 201.0 & 200.0 & 0.63 & $0.7 \mathrm{I}$ & +1.0 & -0.08 & 24.21 & Hind $3.0 ; M a ̈ .11 ; O \Sigma 5 ; M a ̈ . ~ 5$ \\
\hline 1848.49 & 207.2 & 207.8 & 0.66 & 0.70 & -0.6 & -0.04 & 10 & Mä. $3 ;$ Da. $2 ; \mathrm{Da} .1 ;$ Bond $2 ; O \Sigma_{2}$ \\
\hline 1849.54 & 219.3 & $2 \mathrm{I} 6.0$ & 0.64 & 0.66 & $+3 \cdot 3$ & -0.02 & $5 \cdot 4$ & Da. $2.1 ; O \Sigma_{3}$ \\
\hline 1850.59 & 231.5 & 225.6 & 0.54 & 0.60 & +5.9 & -0.06 & 8 & $O \Sigma_{3} ;$ Flt. $2 ;$ Mä. 3 \\
\hline 1851.53 & 237.8 & 2359 & $0.4^{2}$ & 0.53 & +1.9 & -0.11 & 22.19 & Mä. 3.2; Da. $2 ; O \check{\Sigma} 10 ; M \ddot{~} 7.5$ \\
\hline 1852.60 & 2508 & 253.5 & 0.41 & 0.44 & -2.7 & -0.03 & 21.19 & 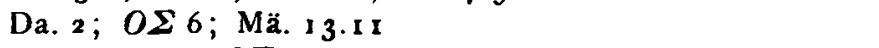 \\
\hline 1853.51 & 270.3 & 272.9 & 0.35 & 0.40 & -2.6 & -0.05 & 17 & Ja. $2 ;$ Mä. $5 ; 0 \Sigma 5 ;$ Da. $4 ;$ Mä. I \\
\hline 854.46 & 304.3 & 296.5 & 0.39 & $0.3^{8}$ & +7.8 & +0.01 & 14.13 & Ja. $3 ;$ Da. $3 ; O \Sigma_{4} ;$ Mä. 4.3 \\
\hline 1855.56 & 326.6 & 321.6 & 0.43 & 0.43 & +5.0 & 0.00 & 19.13 & Sec. $2.0 ;$ Winn. 10.6; Da. I.3;OS 4 ; Mä. 2.0 \\
\hline 1856.47 & 339.1 & 337.7 & 0.49 & 0.50 & +1.4 & -0.01 & 30.25 & Winn. $9.6 ; \mathrm{Da} .1 .3 ;$ Ja. $2 ;$ Winn. $8.4 ;$ Sec. $7 ; O{ }_{3}$ \\
\hline 1857.57 & $351 \cdot 3$ & 350.6 & 0.61 & $0.6 \mathrm{I}$ & +0.7 & 0.00 & 18.16 & Mä. $2.0 ;$ Da. $2 ;$ Sec. $7 ; O \Sigma_{4} ;$ Ja. 3 \\
\hline 1858.54 & $1 \cdot 3$ & 359.0 & 0.73 & 0.70 & $+2 \cdot 3$ & +0.03 & 24.11 & Sec. $3.0 ;$ Dem. 10.0;OZ5; Mä. 6 \\
\hline 1859.52 & $5 \cdot 2$ & 5.6 & 0.74 & 0.79 & -0.4 & -0.05 & I 5.11 & Mä. $4 ;$ Sec. $4.0 ; O \Sigma \Sigma_{4} ;$ Da. 3 \\
\hline 1860.35 & 8.4 & IO.I & 0.87 & 0.86 & -1.7 & +0.01 & 2 & Dawes \\
\hline 1861.58 & 16.1 & I 5.6 & 0.92 & 0.94 & +0.5 & -0.02 & 9 & $O \Sigma_{3} ;$ Mä. 6 \\
\hline $1862.6 \mathrm{I}$ & 19.6 & 19.7 & $\circ 87$ & 1.00 & -0.1 & -0.13 & 19.16 & Winn. $3.0 ;$ Dem. I1; Mä. $3 ; O \Sigma_{2}$ \\
\hline $1863 \cdot 5.3$ & 21.8 & 22.9 & 0.95 & 1.04 & $-\mathbf{I} . \mathbf{I}$ & -0.09 & $19+$ & Den. $1_{3} ; O \Sigma_{4} ;$ Ley. -; Sec. 2 \\
\hline 1864.45 & 26.3 & 25.9 & 0.91 & 1.07 & +0.4 & -0.16 & 12 & Dem. ro; En. 2 \\
\hline 1865.40 & 28.5 & 28.9 & I. I 2 & 1.09 & -0.4 & +0.03 & 23 & En. $5 ; O \Sigma_{3} ;$ Dem. $9 ;$ Da. 3 ; Sec. 2 ; Ley. I \\
\hline 1866.52 & 32.5 & 32.4 & I. 23 & I. Io & +0.1 & +0.13 & 22.21 & Ley. $2 ;$ Dem. 9 ; Sec. 3 ; Harv. $4.3 ; O \Sigma_{4}$ \\
\hline 1867.50 & 33.0 & $35 \cdot 3$ & I.10 & I. 10 & -2.3 & 0.00 & 18.16 & Kn. 3 ; Harv. $3.2 ; O \Sigma_{2}$; Dem. 7 ; Ley. 1.0; Du. I; Winn. I \\
\hline 1868.59 & $37 \cdot 5$ & $3^{8.6}$ & 1.03 & 1.09 & -1.1 & -0.06 & 17 & Dem. $7 ; O \Sigma 5 ;$ Du. $4 ;$ Peirce 1 \\
\hline 1869.57 & 40.7 & 41.6 & 1.03 & 1.06 & -0.9 & -0.03 & 10.9 & Du. 9 ; Ley. 1.0 \\
\hline 1870.45 & 45.1 & 44.6 & 1.07 & 1.04 & +05 & +0.03 & 25.22 & Dem. 8 ; Peirce 4.1 ; Gled. 2 ; Ley. - ; Kn. 1 ; Du. $7 ; 0 \Sigma_{3}$ \\
\hline 1871.51 & 47.1 & $48 \cdot 3$ & 1.06 & 1.00 & -1.2 & +0.06 & $25+$ & Ley. - ; Dem. 8 ; Du. 9 ; Ko. 5 ; Sea. 2 ; Gled. I \\
\hline 1872.47 & 51.2 & 52.0 & 1.00 & 0.96 & -0.8 & +0.04 & $29+$ & Ley. - ; Dem. 9; Fer. 7 ; W. \& S. 1; Du. $7 ; O \Sigma_{5}$ \\
\hline 1873.52 & $55 \cdot 9$ & 56.4 & 1.01 & 0.90 & -0.5 & +0.11 & 22.20 & W. \& S. 3 ; Dem. 8 ; Ley. -; Gled. $5.3 ; O \Sigma_{4} ;$ Du. 2 \\
\hline 1874.47 & 60.5 & $6 \mathrm{I} .0$ & 0.89 & 0.85 & -0.5 & +0.03 & 19.17 & Ley. 2.1; Gled. 3 ; Dem. 8 ; W. \& S. 2.1; $O \Sigma_{4}$ \\
\hline 1875.44 & 67.2 & 66.2 & 0.82 & 0.79 & +1.0 & +0.03 & 23 & Dem. 8 ; Sch. 4 ; Du. II \\
\hline 1876.45 & 71.9 & 72.6 & 0.80 & 0.73 & -0.7 & +0.07 & 31.25 & Dem. 8.2; Hl. 4 ; Ley. $1 ;$ Dem. 9 ; Sch. $5 ; O \Sigma_{4}$ \\
\hline 1877.41 & 77.2 & 79.4 & 0.80 & 0.68 & -2.2 & +0.12 & 30.22 & Cop. 1; Dk. $4.2 ;$ W. \&S. $6.0 ;$ Sch. 5 ; Dem. $9 ;$ Plum. $1 ; O \Sigma_{4}$ \\
\hline 187855 & 89.2 & 89.7 & 0.64 & 0.61 & -0.5 & +0.03 & 26 & $\beta_{1} ;$ Dk. $3 ;$ Dem. $8 ;$ Sch. $9 ; O \Sigma \Sigma_{4} ;$ Pritchett I \\
\hline 1879.53 & 100.5 & 100.2 & 0.55 & 0.57 & +0.3 & -0.02 & 11 & Sch. 7 ; Hall 4 \\
\hline 1880.56 & I I 4.5 & I I 2.5 & 0.54 & 0.54 & +2.0 & 0.00 & 23.20 & Big. 2.0; Dk. 3.2 ; Sch. 6; Jed. $5 ; \beta_{5} ;$ Cop. 2 \\
\hline I 881.44 & 124.7 & 123.9 & 0.51 & 0.53 & +0.8 & -0.02 & I 1.9 & Dk. $2.0 ;$ Hl. 4 ; Sch. $4 ; O \Sigma_{1}$ \\
\hline 1882.49 & 140.7 & 137.8 & 0.54 & 0.53 & +2.9 & $+0.0 \mathbf{r}$ & 23.20 & Dk. $3.2 ;$ Hl. 4 ; Sch. $8 ; O \Sigma_{2} ;$ En. 6.4 \\
\hline 1883.55 & 151.8 & 150.9 & $0.5^{8}$ & 0.55 & +0.9 & +0.03 & $39 \cdot 38$ & Sch. 10; Hall 6; En. 7 ; Per. $7 ; O \Sigma \Sigma_{3} ;$ Jed. 6.5 \\
\hline 1884.54 & 163.5 & 162.5 & 0.60 & $0.5^{8}$ & +1.0 & +0.02 & $33: 24$ & Big. $6.0 ;$ HI. 3 ; Per. 6 ; Sch. 6 ; Pr. $1 ; O \Sigma_{3} ;$ En. 5 ; Sea. 3.0 \\
\hline 1885.46 & 170.6 & 171.7 & 0.63 & 0.62 & $-\mathbf{I} \mathbf{I}$ & +0.01 & 26.23 & Cop. o.1; Hl. 4 ; Sch. 10; Sea. \& Sm. 5.1 ; En. 7 \\
\hline $1886.5^{2}$ & $179 \cdot 3$ & I $8 \mathrm{I.1}$ & 0.68 & 0.66 & -1.8 & +0.02 & $34.3^{2}$ & Hl. 5 ; Per. 4 ; Tar. 3 ; Sm. 3.1; Sch. 11 ; En. 8 \\
\hline $1887 \cdot 5 \mathrm{I}$ & I 86. I & I 89.0 & 0.71 & 0.69 & -2.9 & +0.02 & I 9 & Ho. 1 ; Sch. 15 ; Tar. 3 \\
\hline 1888.54 & 195.8 & 196.5 & 0.65 & 0.71 & -0.7 & -0.06 & 23.22 & Hl. 5 ; Cop. 1.0; Sch. $14 ; O \Sigma_{3}$ \\
\hline 1889.53 & 201.7 & $203 \cdot 7$ & 0.66 & $0.7 \mathrm{I}$ & -2.0 & -0.05 & I I & Hl. 4 ; Sch. 6; OL I \\
\hline
\end{tabular}




\begin{tabular}{|c|c|c|c|c|c|c|c|c|}
\hline$t$ & $\theta_{0}$ & $\theta_{c}$ & $\rho_{0}$ & $\varphi_{c}$ & $\theta_{0}-\theta_{c}$ & $\rho_{0}-\rho_{c}$ & $\mathbf{n}$ & Observers \\
\hline 1890.53 & $209^{\circ} 1$ & $211: 4$ & o."64 & 0.69 & $-2: 3$ & -0.05 & 7.6 & Hl. 6; Big. 1.० \\
\hline 1891.51 & 217.6 & 219.1 & 0.65 & 0.64 & $-\mathrm{I} .5$ & +0.01 & 15 & Hl. 3 ; See 1 ; Sch. 8 ; Maw 3 \\
\hline 1892.50 & 229.1 & 229.1 & 0.61 & 0.58 & 0.0 & +0.03 & 23 & H. C. W. I ; Leav. 2 ; Big. I I; Sch. 6 ; Com. 3 \\
\hline I 893.49 & $244 \cdot 1$ & 241.8 & 0.53 & 0.50 & +2.3 & +0.03 & 18.17 & Maw 1; Sch. 7 ; Leav. 3 ; Big. 7.6 \\
\hline 1894.49 & 261.8 & 259.3 & 0.44 & 0.43 & +2.5 & +0.01 & 7 & Sch. $6 ;$ Big. 1 \\
\hline 1895.51 & 285.9 & 282.7 & 0.37 & 0.38 & +3.2 & $-0.0 I$ & 3.6 & See 0.3 ; Comstock 3 \\
\hline
\end{tabular}

The foregoing table shows that the motion is well represented, and that the present elements will need but very slight corrections. The uncertainty in the period does not surpass 0.1 year, and an alteration of the eccentricity amounting to $\pm 0.0 \mathrm{r}$ is not probable. It seems, however, that there are occasional systematic errors in the angles, and hence it is desirable to continue careful measurement with the view of removing or detecting the cause of these irregularities. It will not be many years before a definitive determination of the elements of this interesting binary can be advantageously undertaken. The following is a short ephemeris for the use of observers.

The University of Chicago, I896 March 24.

$\begin{array}{ccc}t & \theta_{c} & \varphi_{c} \\ 1896.50 & 306.9 & 0.39 \\ 1897.50 & 327.7 & 0.45 \\ 1898.50 & 342.9 & 0.54 \\ 1899.50 & 353.8 & 0.64 \\ 1900.50 & 1.6 & 0.73\end{array}$

In the computation of this orbit Mr. Geo. K. Laze. ton, A. B., graduate student in Physical Astronomy, has rendered assistance which merits my grateful acknowledgement.

\section{Die Regulusbedeckung vom 26. Juni 1895.}

(Mitgetheilt von Prof. F.A.C. Oudemans).

Von dieser Bedeckung sind in Greenwich, Düsseldorf, und Utrecht mit Greenwich berechnet. Vielleicht werden Karlsruhe und Utrecht Eintritt am dunkeln und Austritt am hellen Mondrand beobachtet worden. Ich habe nach der von Herrn Prof. Oudemans in A. N. 1763 vorgeschlagenen Methode die Längenunterschiede von Düsseldorf, Karlsruhe

diese kleinen Grössen (resp. 27, 34 und 20 Minuten) nicht zu stark durch die Fehler des Mondortes beeinflusst.

Die Elemente der Rechnung sind:

\begin{tabular}{|c|c|c|c|c|c|}
\hline Ort & $\mid \begin{array}{c}\text { Angen. Länge } \\
\text { östl. von Greenw. }\end{array}$ & Angen. Breite & Eintritt & Austritt & Quelle \\
\hline ireenwic & $0^{\mathrm{h}} \mathrm{o}^{\mathrm{m}} \mathrm{o}^{\mathrm{s}}$ & $+51^{\circ} 28^{\prime} 38^{n} .1$ & $8^{\mathrm{h}} 4^{\mathrm{m}} 3^{5} .00$ & $8^{h} 54^{w} 57^{s} \cdot 22$ & M. N. LVI, p. I 36 \\
\hline iisseldorf & to 2645 & +511225 & $\begin{array}{lll}8 & 32 & 7.0\end{array}$ & $\begin{array}{llll}9 & 2 & 3 & 27.1\end{array}$ & A. N. 3345 \\
\hline Karlsruhe & $+\circ 333^{6}$ & +49029.6 & 84252.6 & $\begin{array}{lll}9 & 33 & 46.7\end{array}$ & A. N. 3315 \\
\hline Utrecht & to $203^{2}$ & +5259.5 & $\begin{array}{llll}8 & 2 & 3 & 49.1\end{array}$ & $\begin{array}{lll}9 & 15 & 22.3\end{array}$ & A. N. 33 I 2 , bericht. A. \\
\hline
\end{tabular}

Die scheinbaren Oerter von Regulus und des Mondes sind aus dem N. A. interpolirt.

Die (doppelt) berechneten Längen östlich von Greenwich sind :

\begin{tabular}{|c|c|c|c|c|}
\hline Ort & Eintritt & Austritt & Mittel & $\mid \begin{array}{l}\text { Mittel minus } \\
\text { N. A. I } 896\end{array}$ \\
\hline Greenwich & $-0^{\mathrm{h}} \circ^{\mathrm{m}} \quad 3^{5} \cdot 4^{8}$ & $-0^{h} o^{m} 4^{s} \cdot 39$ & $\begin{array}{lll}-0^{h} & 0^{m} & 3^{5} \cdot 93\end{array}$ & $-3^{5} \cdot 93$ \\
\hline sseldorf & to $27 \quad 1.98$ & to $265^{8.32}$ & $\begin{array}{lll}+027 & 0.15\end{array}$ & -5.35 \\
\hline Karlsruhe & to 3334.26 & {$\left[\begin{array}{lll}+0 & 33 & 49.67\end{array}\right]$} & - & -2.24 \\
\hline Utrecht & to 2030.46 & to 2025.90 & to 2028.18 & $-3.5^{2}$ \\
\hline
\end{tabular}

die Längenunterschiede :

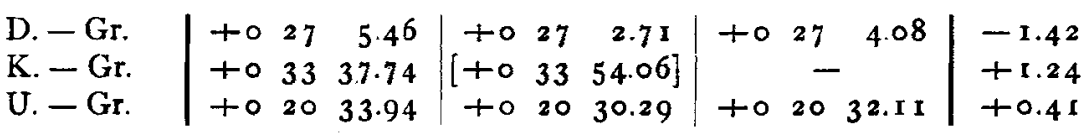

Während die berechneten Längen also alle einen negativen Fehler haben, stimmen die Unterschiede in Länge ziemlich genau. - Den in Karlsruhe beobachteten Austritt, welcher a. a. O. als unsicher angegeben ist, habe ich als wahrscheinlich fehlerhaft ausgeschlossen.

Utrecht 1896 April 29. 